\title{
COMPACT FOURIER ANALYSIS FOR MULTIGRID METHODS BASED ON BLOCK SYMBOLS
}

\author{
THOMAS K. HUCKLE* AND CHRISTOS KRAVVARITIS*†
}

\begin{abstract}
The notion of Compact Fourier Analysis (CFA) is discussed. The CFA allows description of multigrid (MG) in a nutshell and offers a clear overview on all MG components. The principal idea of CFA is to model the MG mechanisms by means of scalar generating functions and matrix functions (block symbols). The formalism of the CFA approach is presented by describing the symbols of the fine and coarse grid problems, the prolongation and restriction, the smoother, and the coarse grid correction, resp. smoothing corrections. CFA uses matrix functions and their features (e.g. product, inverse, adjoint, norm, spectral radius, eigenvectors, eigenvalues), and scalar functions and their roots. This leads to an elementary description and allows for an easy analysis of MG algorithms. A first application is to utilize the CFA for deriving MG as a direct solver, i.e. an MG cycle that will converge in just one iteration step. Necessary and sufficient conditions that have to be fulfilled by the MG components are given for obtaining MG functioning as a direct solver. Furthermore, new general and practical smoothers and transfer operators that lead to efficient MG methods are introduced. In addition, we study sparse approximations of the Galerkin coarse grid operator yielding efficient and practicable MG algorithms (approximately direct solvers). Numerical experiments demonstrate the theoretical results.
\end{abstract}

Key words. multigrid, Fourier analysis, generating function, block symbol, Toeplitz matrices

AMS subject classifications. 65N55, 65F10, 65F15, 65N12, 15A12

1. Introduction. A crucial point for the efficiency of a multigrid (MG) method is the appropriate choice of its components, which allows for an efficient interplay between smoother and coarse grid correction. In many cases, this coordination can be made by use of Local Fourier Analysis (LFA), which is an important quantitative tool for the development of powerful MG methods [3, 24, 23, 13, 4]. This approach has been generalized for structured matrices by employing generating functions expressing, e.g. the symbol of the smoother, of the projection or of the standard discrete Laplace operator in terms of trigonometric polynomials. It is based on the connection between Toeplitz, resp. circulant matrices, and trigonometric functions. It was analyzed by S. Serra Capizzano, R. Chan, T. Huckle, and coauthors in [10, 11, 5, 14, 18, 15, 20, 21]. In this paper we complete this formal approach in order to represent a full two-grid step in terms of the block symbol, called also block generating (matrix) function. Furthermore, we show how to use the block symbol formalism for a multigrid Fourier analysis.

We consider the notion of Compact Fourier Analysis (CFA), which can be seen as a reformulation and generalization of LFA based on matrix functions. Instead of discrete operators on a grid we consider analytic matrix functions (block symbols) of small order that capture the behavior of the full matrices [14]. This allows the use of matrix features, such as product and eigendecomposition, for describing the smoothing factor and the convergence of a multigrid method. CFA offers a more elegant and easier to handle description than the LFA and a clear overview on all MG components. The main properties of a structured matrix can be reduced to a simple function and to its range $[12,15,20]$. We use CFA to

${ }^{*}$ Department of Informatics, Technical University of Munich, Boltzmannstr. 3, 85748 Garching, Munich, Germany (huckle@in.tum.de, kravvarc@in.tum.de).

${ }^{\dagger}$ Supported by Alexander von Humboldt Foundation, Bonn, Germany. 
- derive necessary conditions for smoothing property and convergence expressed as characteristics of the underlying block symbol,

- determine all projections and smoothers that lead to MG as a direct solver,

- identify acceptable projections for an efficient MG method [1],

- design practicable MG components and improved MG algorithms, e.g such that MG acts nearly as direct solver.

It is essential to emphasize that the block symbol reflects in the case of periodic boundary conditions exactly the behavior of the full matrices. For other boundary conditions it mirrors the properties of the full matrices up to a low-rank term. This term is of order $n^{(d-1) / d}$ for $d$-dimensional problems and discretization matrix of size $n$. So in $1 \mathrm{D}$ the low-rank perturbation caused by the boundary conditions is $O(1)$, in 2D $O(\sqrt{n})$, and so on.

The paper is organized as follows. After the necessary introductory material, we describe in sections 1.1 and 1.2 the formalism of CFA with a simple 1D example and explain the connection between discretization stencil of the original PDE and block symbol. Section 1.3 discusses how CFA can be used for determining smoothing factors. Section 1.4 illustrates conditions for the MG convergence in terms of the (block) symbols. Section 1.5 highlights the connection between LFA and CFA, as well as their major differences. Especially, we show how to use CFA for $r$-level Fourier analysis. In section 2 we derive necessary and sufficient conditions for MG functioning as a direct solver. Section 3 is devoted to sparse approximations of the dense Galerkin coarse grid operator, which lead with special combinations of smoother and projection to MG as a nearly, i.e. approximately, direct solver. Section 4 is concerned with numerical experiments based on the block symbol, which verify the theoretical results. In section 5 we consider full V-cycles for the Poisson equation. Section 6 summarizes the conclusions of this study. The appendices at the end aim at offering more insight and understanding on the eigendecomposition of the block symbol, and particular smoothers and projections. It is important to stress that these notions can be defined for multilevel Toeplitz matrices with symbol $f$, and they can be extended also to problems with varying coefficients, indefinite and anisotropic problems.

Notations and preliminary remarks. For simplifying a potential look-up we present some of the notations used repeatedly throughout the paper in the following list.

$$
\begin{array}{ll}
F, f & \text { block and scalar generating symbol of the original problem, resp. } \\
f_{c} & \text { scalar generating symbol of the coarse grid matrix } \\
F^{+} & \text {block symbol of the adjoint matrix of } F \\
B_{R}, B_{P} & \text { block symbol of restriction and prolongation, resp. } \\
b_{1, R}, b_{1, P} & \text { first colum of } B_{R}, B_{P}, \text { resp. } \\
b_{R}, b_{P} & \text { scalar symbol of restriction and prolongation, resp. } \\
M_{R}, M_{L} & \text { block symbol of the pre- and postsmoother, resp. } \\
S_{R}, S_{L} & I-M_{R}^{-1} F, I-M_{L}^{-1} F, \text { resp. } \\
\alpha, \beta & 1+\exp (i x), 1+\exp (i y), \text { resp. } \\
S F, D M & \text { subblock, resp. diagonally modified smoother as rank-1-updates of } F \\
F P & \text { full projection given by } B_{R}=B_{P}=F^{+}
\end{array}
$$

Additionally, the term projection is used for describing both transfer operators if distinction between restriction and prolongation is not necessary or when they are applied symmetrically for defining the coarse grid matrix according to the Galerkin principle as $f_{c}=b_{1}^{H} F b_{1}$. The corresponding scalar generating function and block 
symbol are denoted by $b$ and $B$ (first column $b_{1}$ ). When a differentiation between preand postsmoother is unnecessary or when we refer generally to an explicit smoother, we denote it by $M$. The smoother given by $M$ defines the smoothing iteration $x_{k+1}=$ $x_{k}+M^{-1}\left(b-A x_{k}\right)$ for the iterative solution of a linear system $A x=b$. Similar is the use of the denotation $N$ for approximate inverse smoothers. The smoothers may be considered as approximation $M$ of the matrix $A$ or as approximation $N$ of its inverse.

It holds $F^{-1}=(\operatorname{det} F)^{-1} \cdot F^{+}$, so if the entries of $F$ are trigonometric polynomials, the same is true for $F^{+} . F^{+}$is then also related to a sparse matrix. In the following, square brackets [ ] will be used for stencils and parentheses () for matrices. TGS denotes the block symbol of the matrix describing the full error reduction by a twogrid step. Analogously, $C G C$ stands for the coarse grid correction.

Definition 1.1. A two-grid method is considered to be a direct (i.e. exact) solver if the total error is removed after one iteration.

In other words, it must hold $T G S=S_{L}^{\nu_{l}} \cdot C G C \cdot S_{R}^{\nu_{r}}=0$ (see (1.3) and (1.4)). Evidently it is sufficient that $S_{L}^{\nu_{l}} \cdot C G C=0$ or $C G C \cdot S_{R}^{\nu_{r}}=0$. In this case, the smoother and the projection interact in such a manner that the range of the one matrix is in the nullspace of the other and the actually iterative MG solver degenerates to a non-iterative, direct method. If the coarse system is the same as the original problem up to a constant factor, then the same projection and smoother used on the fine level again make the two-grid method a direct solver on each coarser level, hence the whole MG method can be considered as a direct solver. More informations about MG as a direct solver can be found in $[23,14,4]$.

THEOREM 1.2. [7, p. 72] A circulant matrix $C_{n}$ has the decomposition $C_{n}=$ $F_{n}^{H} \Lambda_{n} F_{n}$, where $\Lambda_{n}$ is the diagonal matrix containing the eigenvalues of $C_{n}$ and $F_{n}$ is the Fourier matrix, which is unitary.

Definition 1.3. Let $\omega=\exp (i \theta)$ with $\theta \in[-\pi, \pi]$. An $n \times n$ matrix $W_{n}$ is called $\omega$-circulant if it has the spectral decomposition $W_{n}=\Omega_{n} F_{n}^{H} \Lambda_{n} F_{n} \Omega_{n}^{H}=\Omega_{n} C_{n} \Omega_{n}^{H}$. $F_{n}$ is the Fourier matrix, $\Lambda_{n}$ is diagonal containing the eigenvalues of $W_{n}, \Omega_{n}=$ $\operatorname{diag}\left(1, \omega^{1 / n}, \ldots, \omega^{(n-1) / n}\right)$ and $C_{n}$ denotes the circulant matrix from Theorem 1.2. An $\omega$-circulant Toeplitz matrix is also characterized by the condition that the last entry in a row and the first entry in the next row differ by the factor $\omega$.

Theorem 1.4 (The Sherman-Morrison formula [6, p. 137]). Let $A$ be a nonsingular matrix and $u, v$ two $n$-vectors with $v^{T} A^{-1} u \neq 1$. Then

$$
\left(A-u v^{T}\right)^{-1}=A^{-1}+\frac{A^{-1} u v^{T} A^{-1}}{1-v^{T} A^{-1} u} .
$$

Model problem. The model problem for describing the block symbol formalism and for the numerical results is the two dimensional Poisson equation

$$
-u_{x x}-u_{y y}=g(x, y)
$$

in the unit square with Dirichlet boundary conditions. The derivatives in (1.1) are replaced by second order finite differences leading to the linear system $A x=b$, where the matrix $A$ is described in compact form by the 5 -point stencil

$$
A_{5}=\left[\begin{array}{ccc} 
& -1 & \\
-1 & 4 & -1 \\
& -1 &
\end{array}\right] \text {. }
$$


Note, that a similar analysis can be performed also for more general stencils like anisotropic or indefinite problems. However, for the sake of simplicity and without loss of generality we focus mainly on (1.1).

1.1. Multigrid and the block symbol formalism: A first example. We want to describe the generating function and the block symbol formalism for the simple 1D Laplacian. The boundary value problem $-u_{x x}=g(x), x \in[a, b], u(a)=$ $u(b)=0$ in standard discretization leads to the stencil $\left[\begin{array}{lll}-1 & 2 & -1\end{array}\right]$ and the matrix $A=\operatorname{tridiag}(-1,2,-1)$. The full weighting restriction operator is related to the stencil $\left[\begin{array}{lll}1 & 2 & 1\end{array}\right]$. The scalar generating functions (called also scalar symbols or simply symbols) to these two stencils are given by $f(x)=-\exp (i x)+2-\exp (-i x)=2(1-\cos (x))$ and $b(x)=2(1+\cos (x))$, respectively [14].

REMARK 1.5. The projection symbol $b(x)$ is closely related to the scaling function in Wavelet theory [22]. The symbol for the scaling function has to have a zero at $\pi$ and the order of this zero determines the approximation quality of the representation. The same holds for the the projection operator $b(x)$ in multigrid. So the symbolic approach used here is similar to the Wavelet formalism. But to deal with fine/coarse grid points on more levels, we have to use a block symbol representation.

The corresponding block symbols can be derived by considering the Toeplitz tridiagonal matrices as block Toeplitz matrices with $2 \times 2$ blocks and related block stencil, e.g. $A=\operatorname{tridiag}(-1,2,-1)$ can be described in block stencil notation as

$$
\left[\left(\begin{array}{cc}
0 & -1 \\
0 & 0
\end{array}\right)\left(\begin{array}{cc}
2 & -1 \\
-1 & 2
\end{array}\right)\left(\begin{array}{cc}
0 & 0 \\
-1 & 0
\end{array}\right)\right]
$$

when standard coarsening is used. This leads to the block generating function

$$
F(x)=e^{i x}\left(\begin{array}{cc}
0 & -1 \\
0 & 0
\end{array}\right)+\left(\begin{array}{cc}
2 & -1 \\
-1 & 2
\end{array}\right)+e^{-i x}\left(\begin{array}{cc}
0 & 0 \\
-1 & 0
\end{array}\right)=\left(\begin{array}{cc}
2 & -\alpha \\
-\bar{\alpha} & 2
\end{array}\right) .
$$

The block symbol related to $b(x)$ is the $e^{i x}$-circulant matrix $B(x)=\left(\begin{array}{ll}2 & \alpha \\ \bar{\alpha} & 2\end{array}\right)$.

From the block symbol $B$ only the first column $b_{1}$ is used to define the projection from fine to coarse grid. Indeed, in the 1D case, there are two classes of grid points in multigrid, which can be modeled by block symbols: grid points that appear also on the coarse level (first index) and grid points that are only fine, but non-coarse (second index). Hence it is clear that coarsening is equivalent to picking only the first column/row in the block symbol.

Also smoothers can be described by block symbols. In the case of the 1D Laplacian, the block symbol of the Jacobi smoother is $\operatorname{diag}(2,2)$ and the Gauss-Seidel smoother is related to the block symbol

$$
\left(\begin{array}{cc}
2 & -e^{i x} \\
-1 & 2
\end{array}\right)=e^{i x}\left(\begin{array}{cc}
0 & -1 \\
0 & 0
\end{array}\right)+\left(\begin{array}{cc}
2 & 0 \\
-1 & 2
\end{array}\right)
$$

which corresponds to the lower triangular part tridiag $(-1,2,0)$. Furthermore, the scalar symbol for the coarse grid problem according to the Galerkin approach is

$$
f_{c}(x)=\left(\begin{array}{cc}
1 & 0
\end{array}\right) B(x) F(x) B(x)\left(\begin{array}{l}
1 \\
0
\end{array}\right)=b_{1}^{H} F(x) b_{1}=4(1-\cos (x))=2 f(x) .
$$

Note that following [10] the symbol of the coarse grid matrix is also given by $f_{c}(x)=$ $\frac{1}{2}\left(b^{2}(x / 2) f(x / 2)+b^{2}(x / 2+\pi) f(x / 2+\pi)\right)$. The error after one two-grid step TGS can 
be analyzed by the error reduction induced by the smoothing operator $S=I-M^{-1} A$, resp. $I-M^{-1} F$ as block symbol, and by the coarse grid correction

$$
C G C=I-P A_{c}^{-1} R^{T} A \text { or } C G C=I-b_{1, P} b_{1, R}^{H} F / f_{c},
$$

in the form

$$
T G S=S_{L}^{\nu_{l}} \cdot C G C \cdot S_{R}^{\nu_{r}}
$$

with postsmoother $S_{L}$ and presmoother $S_{R}$ applied $\nu_{l}$ and $\nu_{r}$ times, respectively. The scalar symbol of the coarse problem for general choice of prolongation and restriction is given according to the Galerkin principle as $f_{c}=b_{1, R}^{H} F b_{1, P}$. The transfer operators can be applied also symmetrically, leading in this case to $C G C=I-b_{1} b_{1}^{H} F / f_{c}$ and $f_{c}=b_{1}^{H} F b_{1}$.

It is easy to see that $b_{1, P}$ belongs to the nullspace of $C G C$, while $b_{1, R}^{H} F$ belongs to the left nullspace of $C G C$.

$$
\begin{gathered}
C G C \cdot b_{1, P}=\left(I-b_{1, P} b_{1, R}^{H} F / f_{c}\right) \cdot b_{1, P}=b_{1, P}-\frac{b_{1, P} b_{1, R}^{H} F}{b_{1, R}^{H} F b_{1, P}} b_{1, P}=b_{1, P}-b_{1, P}=0, \\
b_{1, R}^{H} F \cdot C G C=b_{1, R}^{H} F \cdot\left(I-b_{1, P} b_{1, R}^{H} F / f_{c}\right)=b_{1, R}^{H} F-b_{1, R}^{H} F \frac{b_{1, P} b_{1, R}^{H} F}{b_{1, R}^{H} F b_{1, P}}=0 .
\end{gathered}
$$

1.2. Block symbols and stencils. First we investigate the relationship between PDE, stencil, matrix and block symbol for the general 2D case. The stencil determines the series coefficients of the multilevel Toeplitz, resp. circulant matrix, related to the discretization of the given constant coefficient PDE. Hence, related to the two dimensional stencil

$$
\left[\begin{array}{ccccc} 
& \cdot & \cdot & \cdot & \\
\cdots & a_{1,1} & a_{0,1} & a_{-1,1} & \cdots \\
\cdots & a_{1,0} & a_{0,0} & a_{-1,0} & \cdots \\
\cdots & a_{1,-1} & a_{0,-1} & a_{-1,-1} & \cdots \\
& \cdot & \cdot & \cdot &
\end{array}\right]
$$

is the scalar $2 \mathrm{D}$ symbol

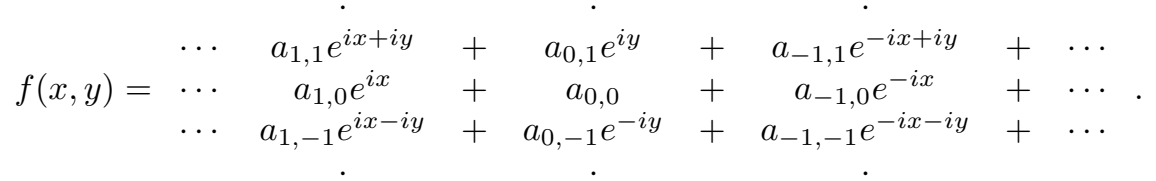

For the block symbol we distinguish between odd and even numbered grid points in each dimension. There are different ways to describe the connection between stencil, matrix, and block symbol. First, like described in [14], we can replace all 1D Toeplitz blocks by block symbols in $x$. This leads to a smaller Block Toeplitz matrix function in $x$. We repeat this first step for this smaller matrix introducing the new variable $y$ and replacing the next block level by block symbols in $x$ and $y$. For a twolevel Block Toeplitz matrix this results in the description of the block symbol by a Fourier series with coefficients given by matrices $F_{j, k}$ in the form

$$
F(x, y)=\sum_{j, k} F_{j, k} \exp (i j x+i k y) .
$$


A second approach can be applied only if the given matrix is a multilevel Toeplitz matrix with known scalar symbol and we want to redefine the matrix as a multilevel Block Toeplitz matrix with block symbol. In Appendix $\mathrm{C}$ we show that in this case the block symbol $F$ is a multilevel $\omega$-circulant matrix. From the scalar generating function $f$ we extract e.g. odd/even scalar coefficients directly to derive the scalar generating functions $F_{j, k}(x, y)$, which are the entries in $F$. These methods are described in [14]. For $2 \mathrm{D}$ with the 5 -point stencil $A_{5}$ we get

$$
F(x, y)=\left(\begin{array}{cccc}
4 & -\alpha & -\beta & 0 \\
-\bar{\alpha} & 4 & 0 & -\beta \\
-\bar{\beta} & 0 & 4 & -\alpha \\
0 & -\bar{\beta} & -\bar{\alpha} & 4
\end{array}\right) .
$$

In the theoretical considerations and numerical experiments in the rest of the paper we use frequently the subblock smoother $S F$ (cf. [14]), which for the model problem (1.1) with discretization stencil $A_{5}$, has block symbol

$$
S F(x, y)=\left(\begin{array}{cccc}
4 & 0 & 0 & 0 \\
-\bar{\alpha} & 4 & 0 & -\beta \\
-\bar{\beta} & 0 & 4 & -\alpha \\
0 & -\bar{\beta} & -\bar{\alpha} & 4
\end{array}\right) .
$$

More informations on how linear systems in the $S F$ smoother can be solved efficiently are given in Appendix A. The smoothing process with $S F$ is closely related to the F-smoothing discussed in [23, Paragraph A.5.1.2].

Additionally, we want to describe the direct connection between block symbol and stencil. In 1D, the stencil is considered as blocks of pairs in the form

$$
\left[\begin{array}{l|lllllllllll}
\cdots & \mid & 0 & -1 & \mid & 2 & -1 & \mid & 0 & 0 & \mid & \cdots
\end{array}\right]
$$

This stencil is related to the block symbol

$$
e^{i x}\left(\begin{array}{ll}
0 & -1
\end{array}\right)+\left(\begin{array}{ll}
2 & -1
\end{array}\right)+e^{-i x}\left(\begin{array}{ll}
0 & 0
\end{array}\right)=\left(\begin{array}{ll}
2 & -\alpha
\end{array}\right) .
$$

In the same way we can write the shifted stencil (applied on the neighboring grid point)

$$
\left[\begin{array}{llllllllllll}
\cdots & \mid & 0 & 0 & \mid & -1 & 2 & \mid & -1 & 0 & \mid & \cdots
\end{array}\right]
$$

with block symbol

$$
e^{i x}\left(\begin{array}{ll}
0 & 0
\end{array}\right)+\left(\begin{array}{ll}
-1 & 2
\end{array}\right)+e^{-i x}\left(\begin{array}{ll}
-1 & 0
\end{array}\right)=\left(\begin{array}{ll}
-\bar{\alpha} & 2
\end{array}\right) .
$$

Combining these two block symbols in a $2 \times 2$ matrix yields the block symbol for $A=\operatorname{tridiag}(-1,2,-1)$. In general, in $1 \mathrm{D}$ the rows of the block symbol $F$ define two - possibly different - stencils, that are related to two typical neighboring rows of the matrix $A$ and to the $2 \times 2$ block structure of $A$.

The same approach can be applied in 2D. The stencil describes a typical row in the matrix. In $2 \mathrm{D}$ with $4 \times 4$ block symbol we could have four different stencils, resp. four typical rows in the discretization matrix $A$ or four different entries in the block symbol. To derive the entries in the block symbol directly, we can use the stencil. But in contrast to the relation of stencil and scalar symbol, cf. (1.7), (1.8), here we 
choose a center point in the stencil (in figure (1.1) there are four independent choices namely entries $4,-1,-1$, and 0 at neighboring positions); around this center point we delete every second entry in each dimension. The resulting stencil can be transformed into a scalar function like before, and this scalar function is one entry in the block symbol.

For the 2D five-point stencil we choose e.g. as center point -1 and define a scalar function by building a Fourier series collecting every second entry as displayed in Figure 1.1. This gives the scalar function $-1-\exp (i x)=-\alpha$. In the same way we get all scalar functions of the block symbol $F$ in (1.9).

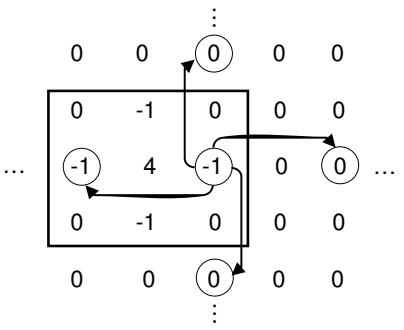

FIG. 1.1. Connection between stencil and block symbol

The block symbols of general multilevel Toeplitz matrices can also be derived very easily in a more technical way. Therefore, we first note that in 1D the block symbol matrix function is defined by appropriate blocking and the related Fourier series as described above. Furthermore, for higher dimensional problems we can set up the scalar symbol directly out of the stencil (see (1.7) and (1.8)). This scalar function can be rewritten as a sum of products of elementary terms, e.g. $\cos \left(j_{1} x\right) \cos \left(j_{2} y\right) \ldots$, where e.g. $\cos \left(j_{1} x\right)$ is related to a $1 \mathrm{D}$ block symbol $F_{1}(x)$. Then, the block symbol for the product is given by the tensor product $F_{1}(x) \otimes F_{2}(y) \otimes \ldots$ In this way, we can set up the block symbol for any scalar symbol, resp. any multilevel Toeplitz matrix, as sum of tensor products of $1 \mathrm{D}$ block symbols.

1.3. Using block symbols for determining smoothing factors. In the scalar case the smoothing property is analyzed by considering the scalar symbol of the smoothing correction $s(x)=1-f(x) / m(x)$ for the high frequency components (e.g. in $1 \mathrm{D} \pi / 2 \leq x \leq \pi$ ). To discuss the smoothing property for the block symbol we consider $S=I-M^{-1} F$ on the subspace related to the nonsingular eigenvalues [14]. Let $u_{j}, j=0, \ldots, k-1$ be the eigenvectors of the $k \times k$ matrix function $F$, related to eigenvalues $\lambda_{j}, j=0, \ldots, k-1 . \quad \lambda_{0}(0)=0$ represents the singularity of $F$ while $\lambda_{j}>0$ and bounded away from 0 for $j=1, \ldots, k-1$. The order $k$ of the block symbol $F$ is defined in dependence on the dimension and the coarsening strategy as $k=k_{d} \cdot k_{t}$, where $k_{d}$ is the dimension of the problem and $k_{t}$ is the ratio of the coarse to fine grid size in one dimension. Note that $\lambda_{0}$, the scalar symbol $f$ and $\operatorname{det} F$ have the same zero of the same order at the origin (see Appendix C). Then, the smoothing property can be determined by considering the projection of $S$ on the subspace spanned by $u_{1}, \ldots, u_{k-1}$, namely the spectral radius of $U_{1}^{H} S U_{1}$ for $x \in[-\pi, \pi]$, where $U_{1}=\left(u_{1} \ldots u_{k-1}\right)$.

In the general case we can analyse the $k \times k$ block symbol $F$ more precisely. As in $1 \mathrm{D}$, cf. (1.2), and as seen in the recursive derivation of the block symbol described in [14], $F$ is - up to a unitary diagonal similarity transform - a multilevel circulant matrix 
(number of levels depending on the dimension, block size depending on the projection: every second, third, ... entry in each dimension). Hence, we can diagonalize $F$ by a unitary diagonal matrix and a multilevel Fourier transform (see also Appendix C).

In $1 \mathrm{D}$ the special block symbol $F$ can be transformed via $D_{x}:=\operatorname{diag}(1, \alpha /|\alpha|)$ into the real circulant matrix

$$
\left(\begin{array}{cc}
2 & -|\alpha| \\
-|\alpha| & 2
\end{array}\right):=C .
$$

So, $F$ is also an 1-level $\omega$-circulant matrix with $\omega=\bar{\alpha} / \alpha=\exp (i x)$. Hence, $C$ can be diagonalized via the Fourier matrix $F_{2}$ of order 2. Then

$$
F=D_{x} F_{2}^{H} \Lambda F_{2} D_{x}^{H}
$$

where $\Lambda$ is a diagonal matrix containing the eigenvalues $\lambda_{0}=f(x / 2)$ and $\lambda_{1}=$ $f(x / 2+\pi)$ of $F$ and $C$.

In $2 \mathrm{D}$ for the 5-point stencil we can apply the diagonal transformation

$$
D=D_{x} \otimes D_{y}=\operatorname{diag}(1, \alpha /|\alpha|, \beta /|\beta|, \alpha \beta /|\alpha \beta|)
$$

for making $F(x, y)$ real two-level circulant. Indeed,

$$
D F D^{H}=\left(\begin{array}{cccc}
4 & -|\alpha| & -|\beta| & 0 \\
-|\alpha| & 4 & 0 & -|\beta| \\
-|\beta| & 0 & 4 & -|\alpha| \\
0 & -|\beta| & -|\alpha| & 4
\end{array}\right) .
$$

This block symbol corresponds to a block circulant matrix with circulant blocks, with eigenvectors given by $U=F_{2} \otimes F_{2}$. The eigenvalues of $F$ and $C$ are then $\lambda_{00}=f(x / 2, y / 2)$, and generally $\lambda_{k j}=f(x / 2+j \pi, y / 2+k \pi), j, k=0,1$. Therefore, $\lambda_{00}$ has to have the same singularity as $f$ and $\operatorname{det} F$ at the origin. The diagonal matrix containing these eigenvalues is in the $2 h$-harmonic LFA the $4 \times 4$ matrix corresponding to the coarse grid system $[23,24]$.

In order to determine the smoothing factor in the block symbol representation, the corresponding block symbols must be diagonalized employing $D$ and $U$. Note, that $D$ and $U$ lead to an explicit representation of the eigenvectors of $F$, e.g. in our $2 \mathrm{D}$ example $u_{j k}, j, k=0,1$. The spectral radius of the matrix function projected on the eigenspace corresponding to the eigenvalues different from $\lambda_{00}$ gives the smoothing factor

$$
\max _{x \in \operatorname{span}\left\{u_{10}, u_{01}, u_{11}\right\},\|x\|=1} x^{H}\left(I-M^{-1} F\right) x .
$$

The analysis of the application of multistage smoothers on both sides can be done similarly by converting the procedure illustrated in [23, Paragaph 7.4.2] into the analogous one with the CFA formalism.

1.4. Necessary Conditions for the projections for feasible MG in terms of the block symbol. In the literature related to algebraic MG [19] and MG for Toeplitz matrices there are described necessary conditions that have to be satisfied by the restriction and prolongation operators, by the smoother, and by the coarse grid problem in order to derive convergence. The following analysis uses the eigendecomposition of the $k \times k$ matrix function $F$ and the different character of eigenvalues: eigenvalue $\lambda_{0} \equiv f\left(\frac{x}{2}, \frac{y}{2}\right)$ with $\lambda_{0}(0,0)=0$ and $\lambda_{j}>0, j=1, \ldots, k-1$. 
TABLE 1.1

Norms and spectral radii of TGS $(x, y)$ for the 5-point stencil and for various multigrid variants with standard Galerkin coarsening, $n=40$ points, presmoother $=G S R B$, postsmoother $=$ subblock

\begin{tabular}{|c|c||c|c|}
\hline restriction & prolongation & norm & spectral radius \\
\hline \hline constant & constant & 0.6979 & 0.4934 \\
\hline standard & trivial & 0.6993 & 0.4943 \\
\hline trivial & standard & 0.0063 & 0.0043 \\
\hline
\end{tabular}

The eigenspace to eigenvector $u_{0}$ is crucial for the coarse grid correction, and the eigenspace to $u_{1}, \ldots, u_{k-1}$ for the smoothing property.

The CGC $k \times k$ block symbol has eigenvalue 1 of order $k-1$ and a single eigenvalue $1-b_{1, R}^{H} F b_{1, P} / f_{c} . F$ has one eigenvalue $\lambda_{0}(\equiv f(x / 2)$ in $1 D)$ with the same singularity at the origin as $f$ and $\operatorname{det} F$. CGC has to reduce the error relative to the singularity, and therefore $\left\|C G C \cdot u_{0}\right\|<\left\|u_{0}\right\|$ must hold, or - with $(1.3)-\left(F u_{0}\right) / f_{c}=\lambda_{0} u_{0} / f_{c}$ has to be bounded and different from zero. Hence, $f_{c}, \lambda_{0}, f$, and $\operatorname{det} F$ have to have the same zeros (note that $\lambda_{0}, f$ and $\operatorname{det} F$ are equal up to a positive trigonometric function).

Rewriting the above condition in terms of scalar symbols we get that

$$
\left\|b_{1} b_{1}^{H} F u_{0} /\left(b_{1}^{H} F b_{1}\right)\right\|=\left\|\lambda_{0} b_{1} b_{1}^{H} u_{0} /\left(b_{1}^{H} F b_{1}\right)\right\|=\left\|\lambda_{0} b_{1}^{H} b_{1} /\left(b_{1}^{H} F b_{1}\right)\right\|=f \cdot f_{c, 1} / f_{c, f}
$$

should be bounded away from 0 and $\infty$. Hereby, $f_{c, g}$ is the coarse symbol derived by applying the projection $b$ with block symbol $B$ and first column $b_{1}$ on function $g$. This gives direct conditions on the zeros of the projection $b$ depending on the zeros of $f$, cf. [1].

In the case of Galerkin coarse grid approach the coarse grid symbol should have the same properties as the original function $f$, and therefore, it must hold

$$
f_{c}=b_{1, R}^{H} F b_{1, P}=f \cdot t
$$

for a trigonometric function $t>0$. Note, that this criterion follows from the condition that the scalar symbol $b_{P} b_{R}$ must have zeros at the so called mirror points at least of the order of the zero 0 of $f$. More information about the mirror points and their importance for appropriate choices of transfer operators in MG can be found in $[11,8]$. So there must not exist an $x$ such that $b_{P} b_{R}$ is zero at $x$ and all its mirror points [11].

Furthermore, it is advisable to derive a CGC block symbol that is well defined and bounded without poles: in the matrix $b_{1, P} b_{1, R}^{H} F / f_{c}$ all coefficients have to be bounded. It is sufficient to assure that $b_{1, R}^{H} F / f_{c}$ is bounded. Thereto we set $b_{1, R}^{H} F / f_{c}=: \tilde{c}^{H}$, where the vector $\tilde{c}$ is bounded and nonzero at the origin. Taking into account that $f_{c}$ equals $\operatorname{det} F$ up to a bounded, strictly positive factor yields the condition

$$
b_{1, R}^{H}=c^{H} F^{+} .
$$

In this case, the block symbol for CGC is bounded for all chosen $b_{1, P}$. Similarly, for

$$
b_{1, P}=F^{+} c
$$

we get that $F \cdot C G C \cdot F^{-1}$ is bounded for each $b_{1, R}$. In both cases $c$ should be welldefined such that $b_{1, R}$ and $b_{1, P}$ have scalar and bounded trigonometric polynomials as entries in order to maintain the sparsity of the related matrices. Therefore, all efficient projections are related to $F^{+}$. For the special case $b_{1}^{H}=c^{H} F^{+}=(1,0, \ldots, 0) F^{+}$the derived projection is the so called full projection FP (see Appendix B). 
In Table 1.1 we see that the choices of restriction and prolongation given in the first two rows do not yield satisfactory convergence properties because the entries in the $C G C$ symbol are not bounded. In this case in the vector $b_{1, R}^{H} F / f_{c}$ the zero of $f_{c}$ is not matched by zeros of appropriate order in all entries of $b_{1, R}^{H} F$. On the contrary, in the third row it is clear that a choice of transfer operators leading to bounded $C G C$ yields more effective two-grid corrections. The similar analysis with the help of scalar generating functions is presented in [1] for general dimensions, where the same conditions on boundedness of scalar functions are obtained.

The requests $(1.10),(1.11)$ and (1.12) are satisfied if $b_{R}$ and $b_{P}$ have zeros at least of the same order as $f$ for $x=0$ at the mirror points and are nonzero at the origin. In general, the scalar symbols $b_{P}$ and $b_{R}$ are allowed to have additional zeros. In [11] the necessary conditions in $2 \mathrm{D}$ for the projection $b$ are described as:

1. $b(0,0) \neq 0, b(0, \pi)=b(\pi, 0)=b(\pi, \pi)=0$, and

2. $b^{2}(x, y)+b^{2}(x, y+\pi)+b^{2}(x+\pi, y)+b^{2}(x+\pi, y+\pi)>0$ for all $(x, y)$.

Considering the block symbol $B(x, y)$ related to $b(x, y)$, if the second condition is violated for a pair $\left(x_{1}, y_{1}\right)$, then this would result in $B\left(2 x_{1}, 2 y_{1}\right)=0$ and therefore cause an additional, not allowed zero in the coarse grid symbol.

1.5. LFA vs. CFA. In this section we would like to point out the strong connection between LFA and CFA, as well as their differences. First we note that both are tools for the quantitative analysis and the design of efficient multigrid methods. An essential difference is that LFA is based on operators on grid functions of the form $\exp (i \theta x / h)$, while CFA deals mainly with matrices of small order, whose entries are scalar generating functions (symbols). CFA employs matrix functions and their features (e.g. product, inverse, adjoint, norm, eigendecomposition, spectral radius), and scalar generating functions and their roots. This characteristic allows for more freedom and easier access with the block symbol.

The procedure described above for calculating smoothing factors with the aid of CFA is equivalent to the smoothing analysis carried out with LFA in [23, Paragraph 4.5]. Both in LFA and CFA, the determination of two-grid convergence factors is reduced to the calculation of norms and spectral radii of $4 \times 4$ matrices (in the $2 \mathrm{D}$ case with standard coarsening). The block symbol is actually an intermediate step between the full matrix and the $2 h$-harmonics LFA matrix. Indeed, given the discretization matrix of a specific problem, one is led to the block symbol according to the strategy described in [14]. The diagonalization of the block symbol as outlined in Appendix C yields the diagonal matrix containing the eigenvalues of the block symbol of the original problem. These eigenvalues correspond to the LFA formal eigenvalues defined in [23, Chapter 4]. With the CFA terminology one can represent the CGC and the TGS, cf. (1.3), (1.4), resp., as matrix functions of small order and accomplish the computations with them. Their norms and eigenvalues coincide with the corresponding LFA results.

Additionally, CFA allows for a discussion about the impact of the coarse grid correction on damping the low-frequency error components. CFA facilitates also the separate analysis and evaluation of projection operators. The corresponding results have been demonstrated in $[10,1]$ in terms of scalar generating functions and their roots. CFA enables the identification of admissible projections for a workable MG method. The study of sparse approximations of the Galerkin coarse grid operator and of sparse approximate inverse smoothers is also possible within the CFA framework.

The advantages of the block symbol are revealed by deploying the formalism to the r-level Fourier Analysis (see [26]). In a first step for the given problem we set up 
the block symbol $F_{r}$ (e.g. in $1 \mathrm{D}$ of size $2^{r-1}$, in $2 \mathrm{D}$ of size $4^{r-1}$ ). Furthermore, all block symbols for the projections on each level have to be defined beforehand. In the case of damped Jacobi smoothing now all symbols for $C G C$ and the smoothing error corrections can be arranged recursively in the standard form

$$
T G S_{2}=S_{L, 2}^{\nu_{L}} \cdot\left(I-\left(b_{P, 2} b_{R, 2}^{H} F_{2}\right) / F_{1}\right) \cdot S_{R, 2}^{\nu_{R}}
$$

and for $l=3, \ldots, r$ :

$$
T G S_{l}=S_{L, l}^{\nu_{L}} \cdot\left(I-b_{P, l}\left(I-T G S_{l-1}\right) F_{l-1}^{-1} b_{R, l}^{H} F_{l}\right) \cdot S_{R, l}^{\nu_{R}} .
$$

The computations can be done, e.g. numerically for an equidistant discretization of the interval $[-\pi, \pi]$. All matrices can be represented in sparse mode, and in the end the matrix norm of $T G S_{r}$ describes one step of the error reduction of the V-cycle operator.

The necessary block symbols of general multilevel Toeplitz matrices can be derived very easily as described in section 1.2. The same technique can be applied for the block symbols, related to the prolongation/restriction, and the Jacobi smoothers. A little bit more complicated is the case of Gauss-Seidel smoothers. To set up the block symbol for these triangular matrices we need on every level the block symbol Fourier series and its coefficient matrices. Therefore, the fine grid block symbol and all projections have to be stored according to their Fourier expansion. For the Galerkin system we have to compute also the Fourier coefficients by building the product of all coefficient matrices according to their position. On each level, out of the Fourier matrix coefficients we can set up any Gauss-Seidel smoother like Red-Black or lexicographically Gauss-Seidel.

The r-level Fourier Analysis can be used as long as $2^{r-1}$, resp. $4^{r-1}$, is not too large, because - depending on the smoother - we have to deal with norms of matrices of size $2^{r-1}$, resp. $4^{r-1}$, that are built from sparse matrices. Therefore, in most cases the maximum matrix size should be less than 1000, or $r \leq 11$ in $1 \mathrm{D}$, resp. $r \leq 6$ in 2D.

2. Deriving multigrid as a direct solver. We aim at deriving a full classification of all two-grid designs based on trigonometric polynomials that are related to MG as a direct solver. In the theoretical proofs of this section we assume that conditions (1.10), (1.11) and (1.12) are satisfied, so that a functioning MG algorithm is feasible. Additionally, for the sake of practicability, the restriction and prolongation matrices must be sparse, i.e. the vectors $b_{1, R}$ and $b_{1, P}$ must have trigonometric polynomials as entries.

2.1. The case of explicit smoother $M$. In this subsection we analyze under what conditions it is possible to derive two-grid as a direct solver, when an explicit smoother $M$ is used. The two-grid direct solver can be extended to an MG direct solver if the coarse grid system is similar to the original, as explained in section 1 after Definition 1.1.

THEOREM 2.1. Let $b_{1, P}$ be a given prolongation. Two-grid is a direct solver, when a presmoother $M_{R}$ is used, if and only if

$$
M_{R}=F+\left(F b_{1, P}\right) d^{H},
$$

where $d$ is an arbitrary vector built of scalar functions so that $M_{R}$ is well defined.

Proof. Suppose that two-grid is functioning as a direct solver. Since $C G C$ has an one-dimensional nullspace, $I-M_{R}^{-1} F$ has to eliminate all the remaining components, 
hence it should be of rank 1. Therefore, we can suppose that $M_{R}-F=c d^{H}$ for arbitrary vectors $c$ and $d$ of appropriate dimensions. According to Theorem 1.4 we have

$$
M_{R}^{-1}=F^{-1}-\frac{F^{-1} c d^{H} F^{-1}}{\gamma},
$$

where $\gamma=1+d^{H} F^{-1} c$. Multiplication by $F$ from the right yields

$$
M_{R}^{-1} F=I-\frac{F^{-1} c d^{H}}{\gamma} \Leftrightarrow I-M_{R}^{-1} F=\left(F^{-1} c\right) \frac{d^{H}}{\gamma} .
$$

Since we presume a direct solver it holds

$$
C G C \cdot\left(I-M_{R}^{-1} F\right)=0 \Leftrightarrow C G C \cdot\left(F^{-1} c\right) \frac{d^{H}}{\gamma}=0 .
$$

Taking into account that $C G C \cdot b_{1, P}=0$ always applies, cf. (1.5), a straightforward choice for prolongation is $b_{1, P}=F^{-1} c$, which implies $c=F b_{1, P}$ and the presmoother is defined as $M=F+\left(F b_{1, P}\right) d^{H}$.

Now suppose $M_{R}=F+\left(F b_{1, P}\right) d^{H}$. Like before, we see that

$$
M_{R}^{-1}=F^{-1}-\frac{F^{-1} F b d^{H} F^{-1}}{1+d^{H} F^{-1} F b}=F^{-1}-\frac{b d^{H} F^{-1}}{1+d^{H} b} .
$$

It holds

$$
C G C \cdot\left(I-M_{R}^{-1} F\right)=\left(I-b b^{H} F / f_{c}\right)\left(I-\left(F^{-1}-\frac{b d^{H} F^{-1}}{1+d^{H} b}\right) F\right)=0,
$$

so the presmoother $M_{R}$ given by (2.1) leads to two-grid as a direct solver $\square$ Similarly we can prove the following Theorem.

THEOREM 2.2. Let $b_{1, R}$ be a given restriction. Two-grid is a direct solver, when a postsmoother $M_{L}$ is used, if and only if

$$
M_{L}=F+c b_{1, R}^{H} F,
$$

where $c$ is an arbitrary vector built of scalar functions so that $M_{L}$ is well defined.

However, the expressions (2.1) and (2.2) for $M$ have the drawback that for bounded $c$, resp. $d, M$ is equally ill-conditioned as $F$ and $M^{-1}$ is as complicated to compute as $F^{-1}$. A possibility to overcome this peculiarity is to introduce the additional constant $\operatorname{det} F$ and to incorporate it appropriately in the above expressions for $M$ via $d$, resp. $c$.

Corollary 2.3. Let $b_{1, R}^{H}=e^{H} F^{+}$and let $c=e \cdot(\operatorname{det} F)^{-1}$ be the vector of Theorem 2.2, where $e$ is a vector with trigonometric polynomial entries, not parallel to $u_{0}$. Then $M_{L}=F+e e^{H}$ leads to two-grid as a direct solver, $M_{L}>0$ and $M_{L}$ is well conditioned.

Proof. From Theorem 2.2 we have that the postsmoother has to be of the form (2.2) for a given restriction $b_{1, R}$ and an arbitrary vector $c$, in order to derive two-grid as a direct solver. Hence

$$
M_{L}=F+c b_{1, R}^{H} F=F+\frac{e}{\operatorname{det} F} e^{H} F^{+} F=F+\frac{e}{\operatorname{det} F} e^{H}(\operatorname{det} F) \cdot F^{-1} F=F+e e^{H}>0 .
$$


The next corollary can be proved in a similar fashion.

Corollary 2.4. Let $b_{1, P}^{H}=F^{+} e$ and let $d=e \cdot(\operatorname{det} F)^{-1}$ be the vector of Theorem 2.1, where $e$ is a vector with trigonometric polynomial entries, not parallel to $u_{0}$. Then $M_{R}=F+e e^{H}$ leads to two-grid as a direct solver, $M_{R}>0$ and $M_{R}$ is well conditioned.

For the choice $e:=e_{1}=(1,0, \ldots, 0)^{H}$ the resulting projection is the full projection FP (see Appendix B), and we define $F+r e_{1} e_{1}^{T}$ as the diagonally modified smoother DM. Hence, in view of Corollaries 2.3 and 2.4, these two components lead to two-grid as a direct solver

REMARK 2.5. The results of Corollaries 2.3 and 2.4 can hold also for non symmetric construction of the smoother, i.e. $M=F+u v^{H}$. For example, the subblock smoother is constructed as $S F=F+u v^{H}$, where $u=\left(\begin{array}{cccc}-1 & 0 & 0 & 0\end{array}\right)^{T}$ and $v=$ $\left(\begin{array}{llll}0 & -\alpha & -\beta & 0\end{array}\right)$.

The subblock smoother combined appropriately with the full projection FP leads to a two-grid direct solver. The full projection was introduced in [14] and can also be described by the scalar symbol

$$
b_{f u l l}(x, y):=f(x, y+\pi) f(x+\pi, y) f(x+\pi, y+\pi) .
$$

Specifically, in Appendix B we prove that the projection related to (2.3) is equivalent to $\mathrm{FP}$ given by $b=e_{1}^{T} F^{+}$.

2.2. The case of approximate inverse smoother $N$. In this paragraph we demonstrate conditions for deriving two-grid as a direct solver, when an approximate inverse smoother $N$ is used. We consider only the case of postsmoother application, since the case of presmoother can be handled absolutely similarly.

Lemma 2.6. Let $b_{1, R}$ be a given restriction. Two-grid with approximate inverse postsmoother $S_{L}=I-N_{L} F$ is a direct solver if and only if

$$
S_{L}=a \cdot b_{1, R}^{H} F,
$$

for some vector a of appropriate dimension. In this case it holds

$$
F^{+}=d \cdot N_{L}+\tilde{a} \cdot b_{1, R}^{H},
$$

where $d=\operatorname{det} F$ and $\tilde{a}=d \cdot a$.

Proof. Suppose that (2.4) holds. For a direct solver with approximate inverse postsmoother it is required $S_{L} \cdot C G C=0$, where $C G C$ is given by (1.3). Indeed, the choice $S_{L}=a \cdot b_{1, R}^{H} F$ leads to $S_{L} \cdot C G C=0$ for any arbitrary vector $a$ of appropriate dimension:

$$
S_{L} \cdot C G C=a b_{1, R}^{H} F\left(I-\frac{b_{1, P} b_{1, R}^{H} F}{b_{1, R}^{H} F b_{1, P}}\right)=a b_{1, R}^{H} F-\frac{a b_{1, R}^{H} F b_{1, P} b_{1, R}^{H} F}{b_{1, R}^{H} F b_{1, P}}=0 .
$$

If two-grid is a direct solver, then $S_{L}$ must have rank one, as explained in the proof of Theorem 2.1. Hence we can suppose that $S_{L}=a \cdot c^{H}$ for arbitrary vectors $a$ and $c$. Considering (1.6) we can select $c=F b_{1, R}$ and the result follows straightforwardly.

For the second part of the enunciation we have $S_{L}=I-N_{L} F=a b_{1, R}^{H} F \Leftrightarrow N_{L}=$ $F^{-1}-a b_{1, R}^{H} \Leftrightarrow N_{L}=\frac{1}{d} F^{+}-a b_{1, R}^{H} \Leftrightarrow F^{+}=d N_{L}+\tilde{a} b_{1, R}^{H}$, where $\tilde{a}=d \cdot a$. 
THEOREM 2.7. An approximate inverse postsmoother leading to two-grid as a direct solver is given by $b_{1, R}^{H}=F^{+} e_{4}$, with $e_{4}=(0,0,0,1)^{T}$, and

$$
\left(\begin{array}{cc}
F_{1}^{-1} & 0_{3 \times 1} \\
0_{1 \times 3} & 0
\end{array}\right)
$$

where $F_{1}$ is the upper left $3 \times 3$ block of the block symbol $F$. In this case, the decomposition (2.5) for the adjoint matrix holds, where $\tilde{a}=\left(-U_{1}^{-1} c, 1\right)^{T}, U_{1}$ is the upper left $3 \times 3$ block of the upper triangular matrix $U$ of the $L U$ factorization of $F, c$ is the upper right $3 \times 1$ column vector of $U$.

Proof. Let $d:=\operatorname{det} F$. We consider the $L U$ factorization $F=L \cdot U$, where $L$ is lower triangular with ones on the diagonal and $U$ is upper triangular. It holds

$$
F F^{+}=L U F^{+}=d I \Rightarrow U F^{+} \equiv\left(\begin{array}{cc}
U_{1} & c \\
0_{1 \times 3} & \gamma
\end{array}\right) F^{+}=d L^{-1}
$$

where $\gamma$ is a scalar and $U_{1}$ is a $3 \times 3$ upper triangular matrix. Multiplication of the last equation from the left by $\left(\begin{array}{cc}U_{1}^{-1} & 0_{3 \times 1} \\ 0_{1 \times 3} & 0\end{array}\right)$ yields

$$
\left(\begin{array}{cc}
I & U_{1}^{-1} c \\
0_{1 \times 3} & 0
\end{array}\right) F^{+}=d\left(\begin{array}{cc}
U_{1}^{-1} & 0_{3 \times 1} \\
0_{1 \times 3} & 0
\end{array}\right) L^{-1} \text {. }
$$

Taking into account that $F^{+}$is of the form (2.5), equation (2.6) can be written as

$$
\begin{aligned}
& \left\{\left(\begin{array}{cc}
I & 0_{3 \times 1} \\
0_{1 \times 3} & 1
\end{array}\right)+\left(\begin{array}{cc}
0_{3 \times 3} & U_{1}^{-1} c \\
0_{1 \times 3} & -1
\end{array}\right)\right\} F^{+}=d\left(\begin{array}{cc}
U_{1}^{-1} & 0_{3 \times 1} \\
0_{1 \times 3} & 0
\end{array}\right) L^{-1} \\
\Leftrightarrow & F^{+}=d\left(\begin{array}{cc}
U_{1}^{-1} & 0_{3 \times 1} \\
0_{1 \times 3} & 0
\end{array}\right) L^{-1}+\left(\begin{array}{c}
-U_{1}^{-1} c \\
1
\end{array}\right)\left(\begin{array}{llll}
F_{41}^{+} & F_{42}^{+} & F_{43}^{+} & F_{44}^{+}
\end{array}\right) .
\end{aligned}
$$

From Lemma 2.6 it is easy to see that

$$
N_{L}=\left(\begin{array}{cc}
U_{1}^{-1} & 0_{3 \times 1} \\
0_{1 \times 3} & 0
\end{array}\right) L^{-1}=\left(\begin{array}{cc}
F_{1}^{-1} & 0_{3 \times 1} \\
0_{1 \times 3} & 0
\end{array}\right) \text { and } b_{1, R}=F^{+} e_{4} .
$$

lead to a two-grid direct solver. $\square$

The corresponding results for the presmoother can be proved analogously.

LEMMA 2.8. Let $b_{1, P}$ be a given prolongation. Two-grid with approximate inverse presmoother $S_{R}=I-N_{R} F$ is a direct solver if and only if

$$
S_{R}=b_{1, P} \cdot a,
$$

for some vector a of appropriate dimension. In this case it holds

$$
\left(I-b_{1, P} \cdot a\right) F^{+}=d \cdot N_{R},
$$

where $d=\operatorname{det} F$.

THEOREM 2.9. An approximate inverse presmoother leading to two-grid as a direct solver is given by $b_{1, P}=F^{+} e_{4}$, with $e_{4}=(0,0,0,1)^{T}$, and

$$
\left(\begin{array}{cc}
F_{1}^{-1} & 0_{3 \times 1} \\
0_{1 \times 3} & 0
\end{array}\right),
$$


where $F_{1}$ is the upper left $3 \times 3$ block of the block symbol $F$. In this case, (2.7) holds for the adjoint matrix, where $b_{1, P}=\left(-U_{1}^{-1} c, 1\right)^{T}, U_{1}$ is the upper left $3 \times 3$ block of the upper triangular matrix $U$ of the $L U$ factorization of $F, c$ is the upper right $3 \times 1$ block of $U, U$ is the upper triangular matrix of the $L U$ factorization of $F$.

REMARK 2.10. The projection $F^{+} e_{1}$ in combination with the smoother defined by the inverse of $F_{3}$, the right lower subblock of $F$ (see Appendix A), leads to a direct solver. This approximate inverse smoother is closely related to the subblock smoother $S F$.

REMARK 2.11. The solution of linear systems in $F_{1}$ has to be done via an iterative solver like the preconditioned conjugate gradient ( $\mathrm{pcg}$ ) algorithm and is similar to solving the subblock smoother SF (see Appendix A).

3. Sparse approximations of the Galerkin coarse grid operator. As already demonstrated, cf. e.g. [4, 13, 23], the MG algorithms with coarsening based on the Galerkin principle may lead to efficient solvers for various choices of MG components. However, this coarsening strategy has the disadvantage that it may lead to coarse grid matrices that become thicker in every next grid, without preserving the sparsity of the initial problem. Possible remedies for this issue have been proposed, cf. e.g. $[2,25]$. Additionally we aim at offering a solution with the help of CFA and at eliminating the drawback of the direct solver, which is its stencil growth. Our goal is to produce sparse matrices on coarser levels via stencil collapsing and to be able to apply then recursively always the same scheme. Naturally, this causes the loss of the direct solver. But we can obtain efficient methods, which work as approximate direct solvers, as we will see in the sequel.

Hence it is interesting to specify sparse approximations of the thick Galerkin coarse grid operator $f_{c}=b^{H} F b$ derived by the full projection $\mathrm{FP}$, that lead to a considerable reduction of the computational complexity and allow to use the same smoothers and projections on every level. It is favorable that the replacement of the coarse grid matrix $A_{c}$ is the same as the original matrix $A$ up to a constant factor and of different dimension. Then the quotient $\frac{f}{f_{c}}$ would be constant. But in general this is not the case. Therefore it is interesting to study sparse approximations of $f_{c}$ of the form $\frac{f}{g}$, where $g$ is the scalar generating function corresponding to a structured sparse matrix $G$, i.e. $g$ is a trigonometric polynomial.

According to the proposed approach, $A_{c}$ is replaced approximately by $G^{-1} \tilde{A}$, where $\tilde{A}$ has the same stencil as $A$ but smaller size. Then on the coarser grid the residual equation $A_{c} e=r$ must be solved, or approximately $G^{-1} \tilde{A} e=r \Leftrightarrow \tilde{A} e=G r$. Hence, in this manner we need to solve on every grid a linear system with a matrix similar to the original matrix, yet of smaller dimension. The right hand side is formed by a cheap matrix-vector multiplication.

The benefit of this approach is that the coarse grid system is similar to the fine and this results in a practicable algorithm. For instance, for the discretization with the 5-point stencil on the fine grid it is known that, when the standard full-weighting projection is employed, all coarse grid matrices are given by the thicker 9-point stencil [13, Paragraph 3.7, Exercise 3.9.4] and [23, Example A.2.1]. The methods developed in the following allow the use of the same matrix on every level.

To that end we calculate $\min _{g}\left\|g-\frac{f}{f_{c}}\right\|$. This is the scalar counterpart of the matrix method proposed in [16] for identifying the modified sparse approximate inverse smoother. As an example we consider $g_{1}(x, y)=a_{0}+a_{1}(\cos x+\cos y)$ and the discretization matrix $A$ described in compact form by the 5 -point stencil $A_{5}$. Let 
$h(x, y):=\cos x+\cos y$. Then we consider

$$
\min _{a_{0}, a_{1}}\left\|g_{1}-\frac{f}{f_{c}}\right\|_{2}^{2}=\min _{a_{0}, a_{1}} \int_{0}^{\pi} \int_{0}^{\pi}\left(a_{0}+a_{1} h-\frac{f}{f_{c}}\right)^{2} d x d y .
$$

This minimization problem for computing the first two Fourier coefficients of $f / f_{c}$ is solved numerically with Maple. A similar approach can be applied as well for approximations of the Galerkin operator with trigonometric polynomials of higher order. We consider also

$$
\begin{aligned}
& g_{2}(x, y)=g_{1}(x, y)+a_{2}(\cos (x-y)+\cos (x+y)), \\
& g_{3}(x, y)=g_{2}(x, y)+a_{3}(\cos 2 x+\cos 2 y) \\
& g_{4}(x, y)=g_{3}(x, y)+a_{4}(\cos (2 x-y)+\cos (x-2 y)+\cos (2 x+y)+\cos (x+2 y))
\end{aligned}
$$

and the constant case $g_{0}(x, y)=a_{0}$. We use the following denotation: trigonometric polynomials of degrees $1,2,3,4,5$ are called the trigonometric polynomials that have the forms $g_{0}, g_{1}, g_{2}, g_{3}, g_{4}$, respectively.

The goal of this approach is furthermore to derive efficient MG methods which work as approximately direct solvers. As approximately direct solvers we declare MG methods that lead to satisfactory enough errors, i.e. of order $10^{-6}$, after application of 2 or $3 \mathrm{~V}$-cycles. The appropriate computer program was developed to this end in a hybrid (i.e. symbolic and numerical) environment. For degrees $d=1, \ldots, 5$ of the approximating trigonometric polynomial and for $k=1,2,3$ applications of the smoothers there has been an exhaustive search over all possible MG components (pre- and postsmoother, restriction, prolongation). The corresponding methods have been tested with respect to the norm and spectral radius of $T G S(x, y)$. For preand postsmoothers there have been examined: subblock smoother, Red-Black GaussSeidel (GS-RB), GS with lexicographic ordering (GS-LEX) and $\omega$-Jacobi $(\omega-J A C)$ with the optimal relaxation parameter $\omega=0.8$. Possible restrictions were: full, standard (full weighting), trivial and constant.

Table 3.1 shows the best results that appeared with respect to spectral radius of $T G S(x, y)$, and also for which restriction. The best results are always obtained with the full prolongation combined appropriately with a standard restriction and with the use of the subblock smoother as pre- and postsmoother. We see that the approximating trigonometric polynomials of degrees $1, \ldots, 4$ yield approximately direct solvers, while the approximation of degree 5 gives significantly better results. These values can always be further improved by multiple applications of the smoothers.

It is also interesting to mention that approximations of the Galerkin coarse grid operator with higher order trigonometric polynomials don't always improve the overall convergence quality. For instance, in Table 3.1 we observe that the increase of the degree from $d=1$ to $d=2$ doesn't lead to an improvement of the results. Similarly, from $d=3$ to $d=4$. The explanation is that with the proposed approach $\min _{g}\left\|g-\frac{f}{f_{c}}\right\|$ is determined, which finds an optimal approximation of $f / f_{c}$, but does not necessarily guarantee that $\min \left\|S_{l} \cdot C G C \cdot S_{r}\right\|$ is attained.

Hence, we can conclude that it is advisable to use sparse approximations of the Galerkin operators of order $d=1,3,5$. For $d=1$ we obtain satisfactory results while preserving at the same time the sparsity of the original problem. For $d=5$ we have the clearly best results, yet with some encumbrance of the sparsity, which is however definitely not lost. An average situation between these two extreme cases is for $d=3$.

REMARK 3.1. The numerical results for our model problem indicate that approximations of the Galerkin coarse-grid operator with trigonometric polynomials $g$ of degree $d=5$ yield always the most efficient solvers. Although $g$ is related in this case 
TABLE 3.1

Optimal spectral radii of $T G S(x, y)$ for various restrictions, full prolongation and subblock preand postsmother, for approximation of the Galerkin coarse grid scalar symbol with trigonometric polynomials of degree $d, n=50$ grid points, $\nu$ is the number of pre-and postsmoother applications.

\begin{tabular}{|c|c||c|c|c|}
\hline$d$ & restriction & $\nu=1$ & $\nu=2$ & $\nu=3$ \\
\hline \hline 1 & trivial & 0.0316 & 0.0315 & 0.0314 \\
\hline 2 & standard & 0.0425 & 0.0423 & 0.0421 \\
\hline 3 & trivial & 0.0127 & 0.0127 & 0.0126 \\
\hline 4 & standard & 0.0155 & 0.0154 & 0.0154 \\
\hline 5 & trivial & 0.0066 & 0.0042 & 0.0031 \\
\hline
\end{tabular}

to a matrix that is less sparse than the coarse grid matrix itself, the practicability is not harmed at all because the proposed approach does not include any explicit matrix inversion. Furthermore, with this technique the sparsity of the stencil describing the problem on coarser levels is preserved.

Instead of approximating the Galerkin coarse function directly in an optimal way, we can use the CFA tool in order to determine trigonometric polynomials $\tilde{g}_{j}$ of degree $j$ that minimize the two-grid error, e.g. in connection with damped Jacobi smoothing

$$
\left(I-\omega_{l} F\right)^{\nu_{l}}\left(I-b_{1} b_{1}^{H} F /\left(f / \tilde{g}_{j}\right)\right)\left(I-\omega_{r} F\right)^{\nu_{r}} .
$$

First let us assume that $\tilde{g}_{j}=\tilde{a}_{0}=$ constant. In order to determine the optimal constant $\tilde{a}_{0}$ we are minimizing the TGS (3.1) for $\omega_{l}=\omega_{r}=0.8, \nu_{l}=\nu_{r}=2$, and $b_{1}$ the full prolongation and restriction. Numerical computations lead to the solution $\tilde{g}_{0}=\tilde{a}_{0}=3.7175 / 64^{2}$. In the same way we can determine the optimal trigonometric polynomials $\tilde{g}_{1}=(2.425+0.7506 \cdot(\cos (x)+\cos (y))) / 64^{2}$ and $\tilde{g}_{2}=$ $(2.6251+0.4624 \cdot(\cos (x)+\cos (y))+0.2020 \cdot(\cos (x-y)+\cos (x+y))) / 64^{2}$. Here, $\tilde{g}_{0}$ leads to 0.0634 as maximum value of the spectral norm of the TGS block symbol (3.1) with full projection, 2 presmoothing steps with SF and 2 postsmoothing steps with optimally damped Jacobi smoother. $\tilde{g}_{1}$ gives error reduction 0.0167 , and $\tilde{g}_{2}$ leads to 0.0106 . We will compare the two different methods based on approximation $f_{c}$, resp. $T G S$, later on in section 5. But note, that this optimization is derived by considering the two-grid analysis. Hence it may fail if considering more levels and should be replaced by an $r$-level analysis as introduced in section 1.5.

4. Experimental Results based on the Block Symbol. Table 4.1 presents for the 2D 5-point stencil the norms and spectral radii of $T G S(x, y)$ for various schemes based on the block symbol. Such computations reflect also the behavior of the full matrices, which is sufficiently well captured by the block symbol [14]. In the following, for pre- and postsmoothing we apply the identical method, and the same holds for restriction and prolongation. The first line shows the results for the standard choice of optimal MG components, which is the use of the Red-Black Gauss-Seidel smoother and of the full weighting projection. In the second line we see that the use of the subblock smoother instead of GS-RB leads to slightly better results. And in the third line it is evident that the combined use of the subblock smoother together with the full projection leads to a direct solver.

REMARK 4.1. The MG components devised and analyzed in this work (cf. also the Appendices) can be exploited and extended also for more general problems, e.g. with nonconstant coefficients:

- The subblock smoother SF is always defined and it is obtained directly from the characterization of fine/coarse points. In the block symbol we replace the 
TABLE 4.1

Norms and spectral radii of TGS(x,y) for various multigrid variants with standard Galerkin coarsening, $n=40$ points.

\begin{tabular}{|c|c||c|c|}
\hline smoother & projection & norm & spectral radius \\
\hline \hline GS-RB & standard & 0.0703 & 0.0311 \\
\hline subblock & standard & 0.0409 & 0.0258 \\
\hline subblock & full & $1.6024 \mathrm{e}-14$ & $1.1269 \mathrm{e}-14$ \\
\hline
\end{tabular}

column (or row) representing the coarse grid points by the diagonal entry only. Hence, in the original matrix A for each coarse grid point a column (or row) has to be replaced by zeros up to the diagonal entry. Similarly, for the DM smoother the diagonal entries related to coarse grid points have to be enlarged by $r>0$.

- The full projection is determined always from the original stencil by sign conversion. For each stencil at a grid point we define the local stencils derived by changing the sign in each second entry. The projection results as a combination of the product of the related matrices and the trivial injection (see Appendix B).

- The approximation of the Galerkin coarse system, e.g. $\min \left\|f_{c}-f / g\right\|$ can be formulated as a sparse matrix problem $\min \left\|A_{c} G-A\right\|_{F}[16]$.

5. Experimental results for $\mathrm{V}$-cycle convergence for the 2D Poisson equation. So far we have examined only the block symbol for the Poisson equation, which served as a model problem for presenting the CFA formalism and features and for testing the new MG components. In this section we will compare the derived MG components for solving the original 2D linear systems for the 5-point stencil by $\mathrm{V}$ cycles. We always use random right hand side of length $\left(2^{7}-1\right)^{2}$ and coarsest matrix size $\leq 8$. We compare standard linear prolongation with full restriction, optimally damped Jacobi smoother with subblock smoother SF defined in section 2.1 and the diagonally modified smoother DM from Corollaries 2.3 and 2.4. Furthermore, for the full projection we consider the different Galerkin coarse system approximations described in section 3. The general benefits of the use of the developed approximate direct solver based on full projection, subblock or diagonally modified smoother, and approximating the Galerkin coarse grid matrix are that less V-cycles are needed. Furthermore, mostly simple matrix-vector products are needed which may lead to better parallel performance.

Following Figure 5.1 we see that the combination of full projection FP, subblock smoother SF and sparsification of the coarse matrix leads to an efficient MG method with faster convergence than standard MG. The additional costs are a more costly projection (around a factor 2 more expensive than full weighting projection), the additional matrix-vector product of the Galerkin approxiamtion matrix $G$ with the actual residual vector. Solving the subblock smoother for the 5-point stencil is comparable to one Jacobi step (see Appendix A). In the new setting the sparse original matrix $A$ is always used on all levels; this simplifies the implementation and saves costs.

Figure 5.2 shows that with the diagonally modified smoother also in combination with standard coarsening we get much faster convergence. Furthermore, with the parameter $r$ we can decide on the number of necessary $\mathrm{V}$-cycle steps.

Furthermore, following Table 5.1 with the choice of $r$ we can distribute the computations between costs for the smoother and number of V-cycle steps. For small $r$ we derive an approximate direct solver with a smoother that has relatively large 


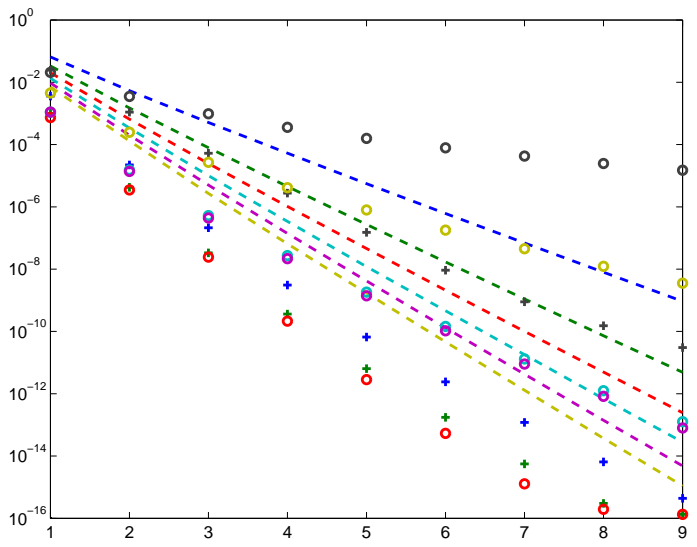

FIG. 5.1. Comparison of the optimally damped standard Jacobi smoother with the subblock smoother combined with full projection and approximation of the coarse Galerkin coarse system. We always apply 2 steps of optimal damped Jacobi postsmoother. Dashed: Jacobi with 2,4,6,8,10,12 presmoothing steps. circle: Approximate direct solver with 2 steps subblock presmoother and trigonometric approximation $g_{j}$ of the coarse grid matrix with 1,2,3,4, or 5 coefficients. + : Approximate direct solver with two steps subblock presmoother and triginometric approximation of the coarse grid matrix based on the TGS block symbol $\tilde{g}_{j}$ with 1,2, or 3 coefficients.

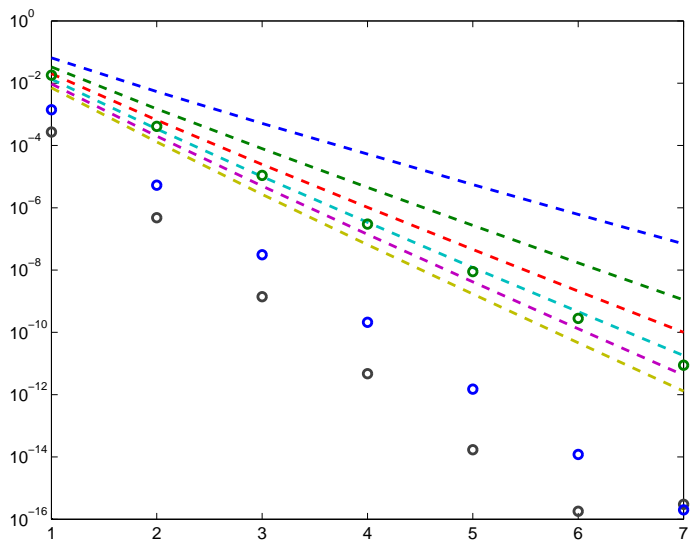

FIG. 5.2. For full weighting projection comparison of standard Jacobi (dashed like Figure 5.1) with the diagonally modified smoother. 2 post/presmoothing steps are applied with $r=4,1,0.5$.

TABLE 5.1

Comparison of different data for the DM smoother. Condition number for different preconditioners, block symbol two-grid error with 2 post/presmoothing steps, and residual after one step of the full V-cycle with full weighting projection and 2 post/presmoothing steps.

\begin{tabular}{|c||c|c|c|c|c|}
\hline$r$ & cond(GS-prec.) & cond(Block-GS-prec.) & smooth. factor & TGS-error & V-cycle residual \\
\hline \hline 0.5 & 13.7 & 8.8 & 0.09 & 0.011 & 0.004 \\
\hline 1 & 7.7 & 4.9 & 0.15 & 0.022 & 0.010 \\
\hline 2 & 4.7 & 3.2 & 0.25 & 0.043 & 0.023 \\
\hline 3 & 3.8 & 3.0 & 0.32 & 0.063 & 0.035 \\
\hline 4 & 3.3 & 3.0 & 0.37 & 0.081 & 0.044 \\
\hline
\end{tabular}


condition number. For larger $r$ we need more V-cycle steps based on an easy to solve smoother.

REMARK 5.1. The smoothers that are used in this section have to be solved by iterative methods, e.g. the preconditioned conjugate gradient method. But such techniques are meanwhile very common in $M G$ theory, see [9]. Furthermore, the smoothers are well conditioned and in many cases we have very efficient preconditioners at hand. Hence, only a small number of iterations is necessary. The cg algorithm applied on $A$ is usually not considered as an efficient smoother because it is too democratic in the sense that it solves also for low-frequency components. By introducing cg applied on the modified problems SF or DM we force cg to work mainly on high frequency components which should improve the smoothing efficiency of the cg method.

6. Conclusions. A compact Fourier analysis was devised for studying MG methods and for designing new efficient MG algorithms. The CFA is principally based on the block symbol. The connection between LFA and CFA is established, however the latter is easier to handle, offers a better overview and is suitable for analyzing all MG components, e.g. by $r$-level Fourier analysis. The block symbol is an intermediate step between the full matrix and the $2 h$-harmonics LFA matrix. Furthermore, the block symbol of the multilevel Toeplitz matrix is a multilevel $\omega$-circulant matrix with eigenvalues given by the scalar symbol.

The CFA, which was first introduced in [14], is elaborated further in this work and more general and theoretical results are given. We present the CFA framework and the necessary conditions in terms of the block symbol that have to be satisfied by the projections for deriving functioning MG methods. We characterize generally the various smoothers that lead to MG as a direct solver in terms of given transfer operators. The results on direct solver hold for every stencil, type of discretization and also for anisotropic and indefinite problems (e.g. Helmholtz equation). The paper develops templates, like the full projection and the subblock smoother, and shows how to incorporate them efficiently in MG. It turns out that the ideal projections are closely related to $F^{+}$, the adjoint of the block symbol $F$ of the original matrix. The ideal smoothers are rank-1 perturbations of $F$.

A procedure for approximating the dense Galerkin coarse grid operator with sparse matrices was developed, leading to practicable approximately direct solvers. The various numerical experiments confirm the theoretical results and the superiority of the use of the full projection and of the subblock smoother, which can be applied successfully also to problems of real-life applications with practical interest.

\section{Appendix A. The subblock smoother.}

Lemma A.1. The lower right $3 \times 3$ block $F_{3}$ of $S F$ is positive definite.

Proof. For the subblock $F_{3}$ holds $F_{3} \geq 0$, since $F \geq 0$. The proof will be done by contradiction. Suppose the $F_{3}$ is singular. This is possible only for the root of the symbol $(x, y)=(0,0)$. In that case there exists an eigenvector $a$ for which $F_{3} a=0$ holds. Then $a$ is part of the eigenvector $b$ of $F$ for which $F b=0$. This eigenvector $b$ corresponds to the eigenvalue $\lambda_{0}=f\left(\frac{x}{2}, \frac{y}{2}\right)$, or that is to say, $b$ is the vector $(1,1,1,1)^{T}$, up to an $\omega$-circulant diagonal transformation. But for the resulting subvector $a$ of length $3 F_{3} a^{H}$ is not 0 , and this is a contradiction.

In order to efficiently use the subblock smoother, we have to solve a linear system with matrix $S F$. To solve a linear system in the subblock smoother we mainly have to solve a linear system in the well conditioned $F_{3}$. For the 5 -point stencil it holds $\operatorname{cond}\left(F_{3}\right)=3+2 \sqrt{2}$. The condition number of the symmetric Gauss-Seidel preconditioned $F_{3}$ matrix is 2 . 
For the solution of linear systems with the subblock smoother we can further reduce the matrix $F_{3}$

$$
\left(\begin{array}{ccc}
4 & 0 & -\beta \\
0 & 4 & -\alpha \\
-\bar{\beta} & -\bar{\alpha} & 4
\end{array}\right)=\left(\begin{array}{ccc}
4 & 0 & 0 \\
0 & 4 & 0 \\
-\bar{\beta} & -\bar{\alpha} & s
\end{array}\right) \cdot\left(\begin{array}{ccc}
1 & 0 & -\beta / 4 \\
0 & 1 & -\alpha / 4 \\
0 & 0 & 1
\end{array}\right)
$$

with Schur complement

$$
s=1-|\alpha|^{2} / 16+|\beta|^{2} / 16=\left(16-|\alpha|^{2}-|\beta|^{2}\right) / 16=(6-\cos (x)-\cos (y)) / 8 .
$$

Therefore, for solving $F_{3}$ we mainly have to solve the linear system in the matrix to scalar symbol $s$ with condition number bounded by 2. With symmetric Gauss-Seidel the condition number for the matrix representing $s$ can further be reduced to 1.5. Hence, in case of the 5-point stencil the cost for smoothing with the subblock matrix is not essentially higher than for other standard smoothers.

\section{Appendix B. The full projection.}

In this paper the full projection $b_{\text {full }}$, which was introduced in [14], is used for deriving new efficient MG methods. An alternative formulation of the Definition 2.3 is that the full projection results for every scalar symbol $f$ from the product of shifted versions of $f$ related to the mirror points. A description of the full projection in matrix form can be found in [17]. The block symbol for the full projection can be described according to Lemma B.1.

LEMma B.1. In 2D with reduction to half size in each dimension, the block symbol of the full projection is $F^{+}$, where $F$ is the block symbol of the original problem.

Proof. For the scalar symbol $f(x, y) \equiv f_{0,0}(x, y)$ in $2 \mathrm{D}$ with projection on every second entry in each dimension, we define the three functions $f_{0,1}(x, y)=f(x, y+\pi)$, $f_{1,0}(x, y)=f(x+\pi, y)$ and $f_{1,1}(x, y)=f(x+\pi, y+\pi)$. Sums and differences of these four scalar functions build the entries in the multilevel $\omega$-circulant block symbol $F(x, y)$ as described in [14]. Furthermore, following Appendix $\mathrm{C}$ this special structure yields $\operatorname{det}(F(x, y))=f_{0,0}(x / 2, y / 2) \cdot f_{0,1}(x / 2, y / 2) \cdot f_{1,0}(x / 2, y / 2) \cdot f_{1,1}(x / 2, y / 2)$.

Let $b_{f u l l}(x, y):=f_{0,1}(x, y) \cdot f_{1,0}(x, y) \cdot f_{1,1}(x, y)$. Then the block symbol for $f_{0,0} \cdot b_{\text {full }}=\operatorname{det}(F(2 x, 2 y))$ is equal to $\operatorname{det}(F(x, y)) \cdot I$, because for functions in $2 x$ and $2 y$ - in view of [14] - the nondiagonal entries in the block symbol are 0 , and the main diagonal entries reproduce the scalar function up to a scaling factor 2 in $x$ and $y$. Therefore it holds $F(x, y) \cdot B_{\text {full }}(x, y)=\operatorname{det}(F(x, y))$, and hence $B_{\text {full }}(x, y)=$ $F^{+}(x, y)$.

\section{Appendix C. Analysis of the block symbol.}

Lemma C.1. The block symbol of a symmetric multilevel Toeplitz matrix with scalar symbol $f(x, y, \ldots)$ a trigonometric polynomial, is a multilevel $\omega$-circulant matrix function $F(x, y, .,,$,$) with eigenvectors depending only on the size of the block symbol$ and given by a diagonally transformed multidimensional Fourier matrix. Furthermore, the eigenvalues are given by evaluating $f(x, y, \ldots)$ at positions $\left(\left(x+2 \pi j_{1}\right) / k\right),(y+$ $\left.\left.2 \pi j_{2}\right) / k, \ldots\right)$ for $j_{1}, j_{2}, \ldots=0,1, \ldots, k-1$.

Proof. First, let us consider the 1D case. Obviously, the block symbol has an $\omega$-circulant structure because blocks that lie outside the central, constant block are inserted with an additional factor $\exp (i j x)$ in view of the Fourier series representation of the block symbol. To prove this result in a rigorous way, in view of the Toeplitz structure of the block symbol $F$ we only have to evaluate the first row of this matrix. The entries of the first row are trigonometric polynomials built from every $k$-th entry 
in the Fourier expansion of the original scalar symbol, starting with coefficients $r=$ $0,1, \ldots, k-1$. Therefore, these entries are given by

$$
e^{i r x / k}\left(f\left(\frac{x}{k}\right)+e^{2 i \pi r / k} f\left(\frac{x+2 \pi}{k}\right)+\ldots+e^{2 i \pi r(k-1) / k} f\left(\frac{x+2 \pi(k-1)}{k}\right)\right) / k
$$

for $r=0,1, \ldots, k-1$. Hence, the resulting Toeplitz matrix is $\omega$-circulant with $\omega=\exp (i x)$ because the last entry in the each row has to be multiplied by $\exp (i x)$ in order to be equal to the first entry in the next row. Therefore, the $\omega$-circulant block symbol is transformed into a circulant matrix by the similarity transform via the diagonal matrix $D_{x}=\operatorname{diag}\left(\exp (i r x / k)_{r=0, . ., k-1}\right)$ (see Theorem 1.2 and Definition 1.3). The eigenvectors of $F(x)$ are columns of the one-dimensional Fourier matrix $F_{k}$ of length $k$ scaled by $D_{x}$.

The eigenvalues of a circulant matrix with first row $c_{0}, \ldots, c_{k-1}$ are given by the trigonometric polynomial $p(x)$ built by these coefficients, evaluated equidistantly at $p(2 \pi i j / k), j=0,1, \ldots, k-1$. Evaluation of this formula gives the eigenvalues $f((x+2 i \pi r) / k), r=0,1, \ldots, k-1$.

The higher dimensional case can be reduced to the 1D case. First note that then the scalar symbol is written as a sum of products of elementary terms $\exp \left(i j_{1} x\right) \exp \left(i j_{2} y\right) \ldots$ which translates in the block symbol as a sum of tensor products of matrix functions $F_{1}(x) \otimes F_{2}(y) \otimes \ldots$ Obviously, each addend in this matrix function itself is a multilevel $(\exp (i x), \exp (i y), .$.$) -circulant matrix diagonalized by the product of the diagonal$ matrix $D_{x} \otimes D_{y} \otimes \ldots$ and the appropriate multidimensional Fourier matrix. We can apply this eigenvector matrix on the original block symbol given by this sum of tensor products in order to diagonalize $F$. This leads to the diagonal matrix of eigenvalues of $F$ written as sum of tensor products of $1 \mathrm{D}$ diagonal matrices in $x, y, \ldots$, resp.. Therefore, all eigenvalues can be written in the form $g\left(\left(x+2 \pi j_{1}\right) / k,\left(y+2 \pi j_{2}\right) / k, \ldots\right)$ with some function $g$. Furthermore, the first eigenvalue $g(x / k, y / k, .$.$) is related to$ the first column of all ones of the Fourier matrix. Like in the 1D case the sum over all entries in the first row of $F$ results in $f(x / k, y / k, \ldots)$ because all shifted terms cancel out in this summation. This shows $f=g$ which concludes the proof. $\square$

In view of the eigenvalues of $F$ it also holds

$$
\left.\operatorname{det}(F)=\prod_{j_{1}, j_{2}, . .,=0}^{k-1} f\left(\left(x+2 \pi j_{1}\right) / k\right) f\left(y+2 \pi j_{2}\right) / k\right) \ldots
$$

\section{REFERENCES}

[1] A. Aricò And M. Donatelli, A V-cycle Multigrid for Multilevel Matrix Algebras: proof of Optimality, Numer. Math., 105 (2007), pp. 511-547.

[2] M. Bolten And A. Frommer, Structured grid AMG with stencil collapsing for d-level circulant matrices, Preprint BUW-SC 07/4, Bergische Universität Wuppertal, 2007.

[3] A. Brandt, Multi-level adaptive solutions to boundary-value problems, Math. Comp., 31 (1977), pp. 333-390.

[4] W. L. Briggs, V. E. Henson and S. F. McCormick, A Multigrid Tutorial, SIAM, Philadelphia, 2000.

[5] R. Chan, Q. Shang and H. Sun, Multigrid Method for Ill-Conditioned Symmetric Toeplitz Systems, SIAM J. Sci. Comput., 19 (1998), pp. 516-529.

[6] B. N. Datta, Numerical Linear Algebra and Applications, 2nd ed., SIAM, Philadelphia, 2010.

[7] P. Davis, Circulant matrices, John Wiley, New York, 1979.

[8] M. Donatelli, An algebraic generalization of local Fourier analysis for transfer operators in multigrid based on Toeplitz matrices, Numer. Lin. Alg. Appl., 17 (2010), pp. 179-197.

[9] H. C. Elman, O. G. Ernst and D. P. O'Leary, A Multigrid method enhanced by Krylov Subspace Iteration for Discrete Helmholtz Equations, SIAM J. Sci. Comput., 23 (2001), pp. 1291-1315. 
[10] G. Fiorentino And S. Serra, Multigrid methods for Toeplitz matrices, Calcolo, 28 (1991), pp. 283-305.

[11] G. Fiorentino And S. Serra, Multigrid methods for symmetric positive definite Block Toeplitz matrices with nonnegative generating functions, SIAM J. Sci. Comput., 17 (1996), pp. $1068-1081$.

[12] U. Grenander and G. Szegö, Toeplitz forms and their applications, Chelsea Publishing, New York, 1984.

[13] W. HackBusch, Multi-Grid Methods and Applications, Springer, Berlin, 1985

[14] T. K. Huckle, Compact Fourier Analysis for designing Multigrid Methods, SIAM J. Sci. Comp., 31 (2008), pp. 644-666.

[15] T. K. Huckle and D. Noutsos, Preconditioning block Toeplitz matrices, Electron. Trans. Numer. Anal., 29 (2007), pp. 31-45.

[16] T. Huckle and M. SedlaceK, Smoothing and Regularization with Modified Sparse Approximate Inverses, J. Electr. Comput. Eng., Volume 2010, Article ID 930218, 16 pages.

[17] T. Huckle and J. Staudacher, Matrix Multilevel Methods and Preconditioning, BIT, 42 (2002), pp. 541-560.

[18] T. Huckle And J. Staudacher, Multigrid methods for block Toeplitz matrices with small size blocks, BIT, 46 (2006), pp. 61-83.

[19] J. Ruge and K. StüBen, Algebraic Multigrid, in Frontiers in Applied Mathematics: Multigrid Methods, S. Mccormick, (ed.), SIAM, Philadelphia, 1987, pp. 73-130.

[20] S. SERRA, Spectral and computational analysis of block Toeplitz matrices having nonnegative definite matrix-valued generating functions, BIT, 39 (1999), pp. 152-175.

[21] S. Serra and C. Tablino Possio, Multigrid methods for multilevel circulant matrices, SIAM J. Sci. Comput., 26 (2004), pp. 55-85.

[22] G. Strang, Wavelets and Dilation Equations: A Brief Introduction, SIAM Review, 31 (1989), pp. 614-627.

[23] U. Trottenberg, C. W. Oosterlee and A. Schüller, Multigrid, Acad. Press, San Diego, 2001.

[24] R. Wienands and W. Joppich, Practical Fourier analysis for multigrid methods, Chapman \& Hall/CRC, Boca Raton, 2005.

[25] R. Wienands And I. YAvneH, Collocation coarse approximation (CCA) in multigrid, SIAM J. Sci. Comp., 31 (2009), pp. 3643-3660.

[26] R. Wienands and C. W. Oosterlee, On Three-Grid Fourier analysis for Multigrid, SIAM J. Sci. Comp., 23 (2001), pp. 651-671. 